\title{
Elastic and plastic deformation of graphene, silicene, and boron nitride honeycomb nanoribbons under uniaxial tension: A first-principles density-functional theory study
}

\author{
M. Topsakal ${ }^{1}$ and S. Ciraci ${ }^{1,2}$ \\ ${ }^{1}$ UNAM-Institute of Materials Science and Nanotechnology, Bilkent University, Ankara 06800, Turkey \\ ${ }^{2}$ Department of Physics, Bilkent University, Ankara 06800, Turkey
}

(Received 29 November 2009; published 19 January 2010)

\begin{abstract}
This study of elastic and plastic deformation of graphene, silicene, and boron nitride (BN) honeycomb nanoribbons under uniaxial tension determines their elastic constants and reveals interesting features. In the course of stretching in the elastic range, the electronic and magnetic properties can be strongly modified. In particular, it is shown that the band gap of a specific armchair nanoribbon is closed under strain and highest valance and lowest conduction bands are linearized. This way, the massless Dirac fermion behavior can be attained even in a semiconducting nanoribbon. Under plastic deformation, the honeycomb structure changes irreversibly and offers a number of new structures and functionalities. Cagelike structures, even suspended atomic chains can be derived between two honeycomb flakes. Present work elaborates on the recent experiments [C. Jin, H. Lan, L. Peng, K. Suenaga, and S. Iijima, Phys. Rev. Lett. 102, 205501 (2009)] deriving carbon chains from graphene. Furthermore, the similar formations of atomic chains from BN and Si nanoribbons are predicted.
\end{abstract}

DOI: 10.1103/PhysRevB.81.024107

PACS number(s): 62.25.-g, 61.46.-w, 61.48.De, 73.63.-b

\section{INTRODUCTION}

For last two decades honeycomb structured materials have dominated nanoscience. The unique orbital symmetry of the honeycomb structure underlies several exceptional properties of carbon-based nanomaterials, such as fullerenes, nanotubes, graphene, and its quasi-one-dimensional ribbons. While $\pi$ orbitals are responsible for the unusual electronic and magnetic properties of graphene, ${ }^{1,2}$ its planar flexibility but high in-plane strength is achieved by $s p^{2}$-hybrid orbitals. For example, charge carriers in graphene behave like a massless Dirac fermions ${ }^{3}$ due to electron and hole bands showing linear crossing at the Fermi level. These bands are derived from $\pi$ and $\pi^{*}$ states. An unpaired $\pi$ state leads to a local magnetic moment in a nonmagnetic honeycomb structure. ${ }^{4}$ Also the strong overlap between nearest-neighbor $\pi$ orbitals assures the planar stability of graphene and boron nitride (BN). Silicene, a honeycomb structure of Si, lacking such an overlap is stabilized only by puckering. ${ }^{5}$

The recent spectroscopy techniques to identify and quantify the strain profiles in graphene have shown that the Raman peaks shift considerably under the in-plane strain ${ }^{6}$ providing a fundamental tool for graphene-based micromechanical/nanomechanical systems. Some theoretical studies ${ }^{7,8}$ have also shown that local and uniform strain can be an effective ways of tuning the electronic structure and transport characteristics of graphene devices to generate confined states, quantum wires, and collimation. A related step was given by Kim et al., ${ }^{9}$ who have developed a simple method to grow and transfer high-quality stretchable graphene films on a large scale using chemical-vapor deposition on nickel layers, which might enable numerous applications including use in large-scale flexible, stretchable, foldable transparent electronics.

This study investigates the stretching of quasi-onedimensional nanoribbons (NRs) of graphene, BN, and silicene and predicts that they attain new functionalities by changing to a number of new structures with interesting electronic and magnetic properties. While the elastic deformation with harmonic and anharmonic ranges and sudden yielding points are common to all NRs, the absence of sequential elastic deformation stages ending with order-disorder structural transformation each leading to stepwise necking constitutes their prime difference from metal nanowires. ${ }^{10,11}$ Cage structures of large polygons can be generated. In particular, atomic chains of $\mathrm{C}, \mathrm{BN}$, and $\mathrm{Si}$ between honeycomb flakes can form under certain circumstances. The synthesis of these ultimate one-dimensional atomic chains, which can form various stable geometries, ${ }^{12}$ are expected to be an essential step toward future nanotechnology applications. We believe that our predictions are relevant for the current studies investigating the properties of strained graphene.

\section{MODEL AND METHODOLOGY}

We have performed first-principles plane-wave calculations within density-functional theory (DFT) using projectoraugmented wave potentials. ${ }^{13}$ The exchange correlation potential has been approximated by generalized gradient approximation using PW91 (Ref. 14) functional both for spin-polarized and spin-unpolarized cases. Recently, spinpolarized calculations within DFT have been carried out successfully to investigate magnetic properties of vacancy defects in two-dimensional (2D) honeycomb structures. Also interesting spintronic properties of nanoribbons have been revealed using spin-polarized DFT. ${ }^{15}$ The success of spinpolarized DFT calculations has been discussed in Ref. 16.

All structures have been treated within supercell geometry using the periodic boundary conditions. A plane-wave basis set with kinetic-energy cutoff of $400 \mathrm{eV}$ has been used. The interaction between monolayers in adjacent supercells is examined as a function of their spacing. Since the total energy per cell has changed less than $1 \mathrm{meV}$ upon increasing the spacing from 10 to $15 \AA$, we used the spacing of $\sim 10 \AA$ in 
(a)
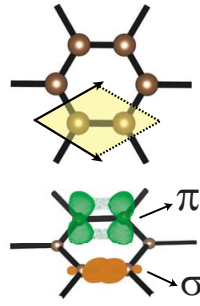

(b)

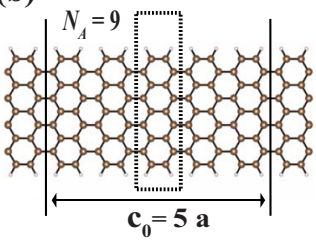

(c)

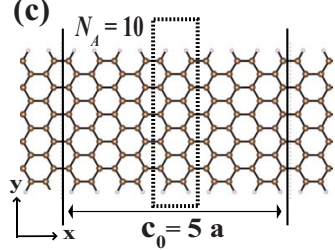

(d)

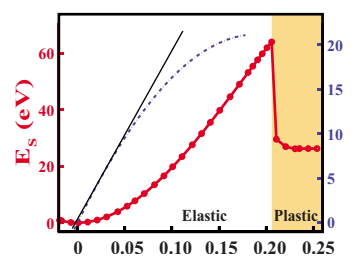

Strain $\varepsilon\left[\Delta \mathrm{c} / \mathrm{c}_{0}\right]$
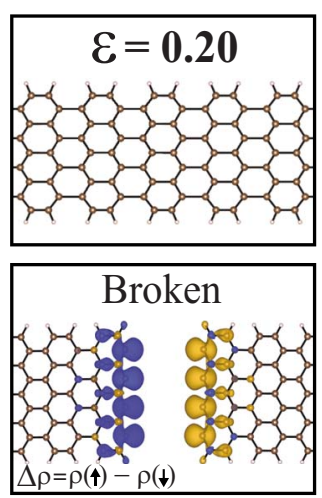

(e)

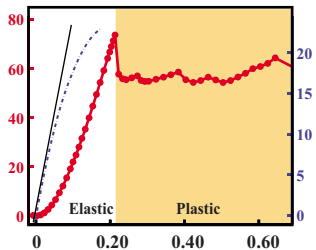

Strain $\varepsilon\left[\Delta \mathrm{c} / \mathrm{c}_{0}\right]$
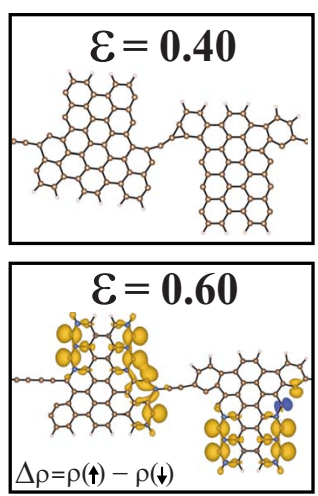

(f)

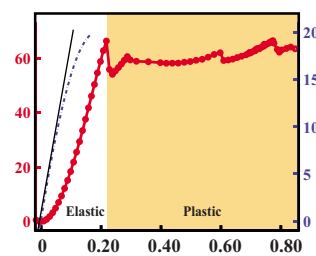

Strain $\varepsilon\left[\Delta \mathrm{c} / \mathrm{c}_{0}\right]$
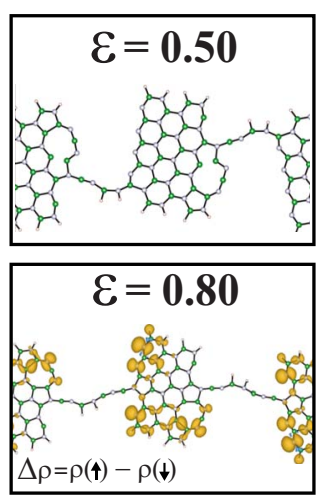

(g) ASNR(10)

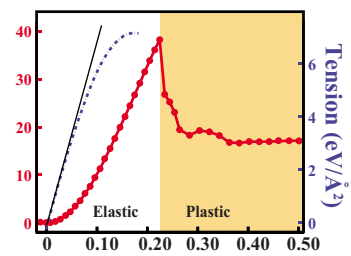

Strain $\varepsilon\left[\Delta \mathrm{c} / \mathrm{c}_{0}\right]$
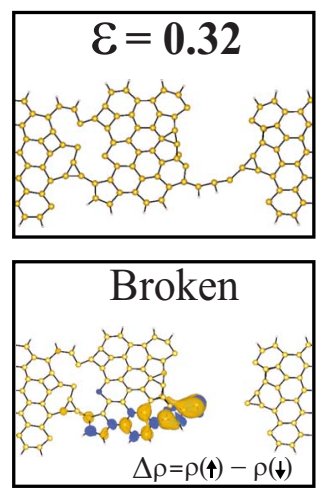

FIG. 1. (Color online) (a) Two-dimensional honeycomb structure with primitive unit cell. $\pi$ and $\sigma$ bonds are schematically described. (b) An armchair graphene nanoribbon AGNR(9) has mirror symmetry with respect to its axis and $N_{A}=9$ dimer bonds across the unit cell specifying its width. The unit cell is delineated by a rectangle with dotted edges. The supercell consists of five unit cells with $c_{0}=5 \times a$. (c) Same for AGNR(10), which lacks the mirror symmetry. (d) Response of AGNR(9) to uniaxial tension examined in the supercell; variation in the strain energy $E_{S}$ and tension force $F_{T}$ with shaded region indicating the plastic range; atomic structure for $\epsilon=0.20$ just before breaking. Isosurfaces for the difference of spin-up (blue/dark) and spin-down (yellow/light) charge densities, $\Delta \rho=\rho(\uparrow)-\rho(\downarrow)$ calculated for broken pieces show antiferromagnetic order of zigzag edge states.(e) Same for AGNR(10). Monatomic carbon chains connect magnetic pieces. (f) Armchair boron nitride NR. (g) Armchair silicene NR.

the calculations. In the self-consistent potential and totalenergy calculations the Brillouin zone is sampled in $\mathbf{k}$ space using Monkhorst-Pack scheme by $25 \times 1 \times 1$ for nanoribbons. This sampling is scaled according to the size of superlattices. All atomic positions and lattice constants are optimized by using the conjugate gradient method, where the total energy and atomic forces are minimized. The convergence for energy is chosen as $10^{-5} \mathrm{eV}$ between two steps. Numerical plane-wave calculations are performed by using VASP package. ${ }^{17,18}$

\section{ELASTIC AND PLASTIC DEFORMATION OF NANORIBBONS}

Nanomechanics of both armchair and zigzag NRs of graphene, silicene, and $\mathrm{BN}$ is explored by calculating the mechanical properties as a response to stretching along the axis of the ribbon. Mechanical properties are revealed from the strain energy $E_{S}=E_{T}(\epsilon)-E_{T}(\epsilon=0)$; namely, the total energy at a given axial strain $\epsilon$ minus the total energy at zero strain. Segments of quasi-one-dimensional NRs are treated within supercell geometry using periodic boundary conditions. Each supercell contains $n$ unit cells of the ribbon and hence has the lattice constant along the axis of ribbon, $c_{0}$ $=n a, a$ being lattice constant for the primitive unit cell of NR. The strain, $\epsilon=\Delta c / c_{0}$, corresponds to a stretching, where the lattice constant of the strained supercell equals $c=c_{0}$ $+\Delta c$. The stretching of the ribbon is achieved by first in- creasing the optimized lattice constant in increments of $\Delta \epsilon$ $=0.01$ (namely, $c \rightarrow c+\Delta \epsilon \times c_{0}$ ) and by uniformly expanding the atomic structure obtained from previous optimization. Subsequently the atomic structure is reoptimized keeping the increased lattice constant $c$ fixed and the corresponding strain energy is calculated. This process is repeated after each increment of $\Delta \epsilon$. Then the tension force, $F_{T}=$ $-\partial E_{S}(\epsilon) / \partial c$ and the force constant $\kappa=\partial^{2} E_{S} / \partial c^{2}$ are obtained from the strain energy. Owing to ambiguities in defining the Young's modulus of 2D honeycomb structures, one can use in-plane stiffness, $C=\left(1 / A_{0}\right)\left(\partial^{2} E_{S} / \partial \epsilon^{2}\right) \cdot{ }^{19,20}$ Here $A_{0}$ is the equilibrium area of the supercell. The in-plane stiffness can be deduced from $\kappa$ by defining an effective width for the ribbon.

The variation in strain energy, tensile force, and the corresponding atomic structure of selected armchair NRs are presented in Fig. 1 as a function of $\epsilon$. Here the bonds parallel to the direction of applied tension is stretched more than those in other directions. As a result, the hexagonal symmetry is disturbed but overall honeycomblike structure is maintained. The elastic deformation is reversible and stretched ribbons can return to their original geometry when the tension is released. In the harmonic range, the force constant is calculated to be $\kappa=176,30$, and $144 \mathrm{~N} / \mathrm{m}$, for armchair graphene, silicene, and BN NRs having $N_{A}=10$, respectively. Similarly, the calculated in-plane stiffness for the same ribbons are, respectively, $C=292,51$, and $239 \mathrm{~N} / \mathrm{m}$. Notably, $\kappa$ and $C$ values of silicene are lowest due to $s p^{2}$ orbitals dehybridized as a result of puckering. Cohesive energies of 
graphene, silicene, and $\mathrm{BN}$ nanoribbons with $N_{A}=10$ (namely, 19.77, 10.76, $18.80 \mathrm{eV}$ per atom pair, respectively) show trends similar to the corresponding in-plane stiffness values. We also calculated the in-plane stiffness values of $2 \mathrm{D}$ graphene, silicene, and $\mathrm{BN}$ honeycomb structures to be, respectively, $C=335$ (the reported experimental value ${ }^{21}$ for graphene: $C=340 \pm 50$ ), 62 , and $258 \mathrm{~N} / \mathrm{m}$. Because of edge effects of NRs, their stiffness values are relatively smaller than those of $2 \mathrm{D}$ structures.

The elastic range ends at the yielding point with the corresponding critical strain,, where the strain energy drops suddenly. It should be noted that $\epsilon_{Y}$ and plastic deformation of nanoribbons are expected to depend on the ambient temperature, unit-cell size, crystalline defects such as vacancy and time rate of change in stretching. The stochastic nature of deformation is avoided to some extent by carrying out a slow or "adiabatic" stretching as explained above, whereby the ribbon is stretched in small increments and the structure is optimized after each increments in elastic and plastic ranges. The stretching of nanoribbon is carried out at $0{ }^{\circ} \mathrm{K}$ for three different values of $\Delta \epsilon$, namely, $0.05,0.01$, and 0.002 . The value of $\epsilon_{Y}$ did not changed for $\Delta \epsilon=0.01$ and 0.002 . We therefore concluded that already $\Delta \epsilon=0.01$ corresponds to a very slow or adiabatic stretching and reveals the bare response of nanoribbon without the effect of time rate of change of stretching. The effect of temperature is investigated by performing $a b$ initio molecular-dynamic calculations (lasting $2 \mathrm{ps}$ with time steps of $2 \times 10^{-15} \mathrm{~s}$ ) for $N_{A}$ $=10$. Results indicate that $\epsilon_{Y}$ decreases with increasing temperature. Hence, owing to the softening of acoustical phonons, $\epsilon_{Y}=0.22$ corresponding to $T=0 \mathrm{~K}$ is reduced to 0.16 at $T=600 \mathrm{~K}$. We also found that the results are converged if the supercell size $n \geq 5$. The presence of a vacancy defect in the ribbon speeds up the yielding by decreasing the value of $\epsilon_{Y}$.

The plastic deformation stage following the yielding point is the crucial part in the stretching of NRs having honeycomb structure and hence is the focus of this work. The response of the ribbon to the strain after the yielding point is material and geometry specific. Having determined various effects, which possibly change the value of $\epsilon_{Y}$, we examine the structure in the range of plastic (irreversible) deformation through adiabatic stretching. The armchair graphene NR with $N_{A}=9$, i.e., $\operatorname{AGNR}(9)$ has a mirror symmetry relative to its $x$ axis. This NR is broken into graphene patches having equilibrium honeycomb structure just after $\epsilon_{Y} \cong 0.21$ in Fig. 1(a). Whereas the behavior of AGNR(10) (which lacks the mirror symmetry) is dramatically different. The ribbon is torn into two pieces (patches), which are connected by an atomic chain. In the plastic range, the strain energy $E_{S}$ increases slightly with strain, but eventually decreases each time when a $s p^{2} \mathrm{C}-\mathrm{C}$ bond of the zigzag edge of the patch is broken and a $\mathrm{C}$ atom is incorporated into the chain from the graphene patch. This important result actually predicts the recent finding by Iijima and his collaborators, who derived monatomic carbon chain from graphene. ${ }^{22}$ Carbon atomic chains identified as cumulene (having double bonds) or polyyne (consisting of alternating triple and single bonds) have been studied earlier. ${ }^{12,23,24}$ The chain structure was found to be stable and linear owing to the strong overlap of $\pi$ orbitals between adjacent atoms. While infinite chain is subject to a Peierls distortion, bond alternation and bond length variation depends on the number of carbon atoms in a finite carbon chain. The character of the covalent $s p+\pi$ bonding between carbon atoms underlies their unusual chemical, mechanical, and quantum transport properties.

Our results show that the tearing of the armchair graphene $\mathrm{NR}$ and hence the formation of a carbon chain is promoted by a vacancy defect. Even more surprising is that not only graphene but also puckered silicene and flat BN armchair ribbons are plastically deformed to form atomic chains between patches. Since BN honeycomb structure is already synthesized, the present results concerning the formation of $\mathrm{BN}$ chains is important and hence is yet to be realized experimentally. In contrast to graphene, pieces (patches) torn from the BN and silicene ribbons allow also different types of polygons ranging from trigon to heptagon. In particular, a large hole in the silicene pieces is reminiscent of the cage structure as if a 2D analog of metal-organic frameworks (a)

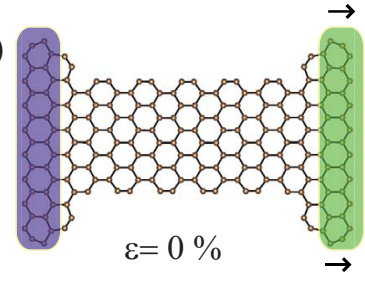

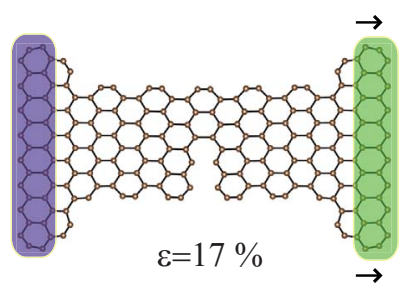

(b)

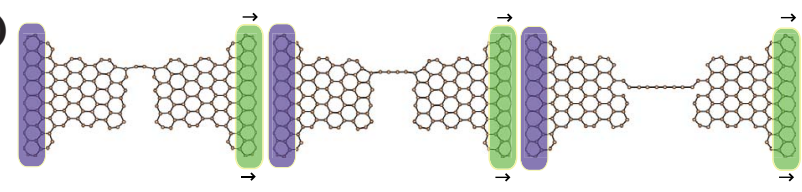

(c)

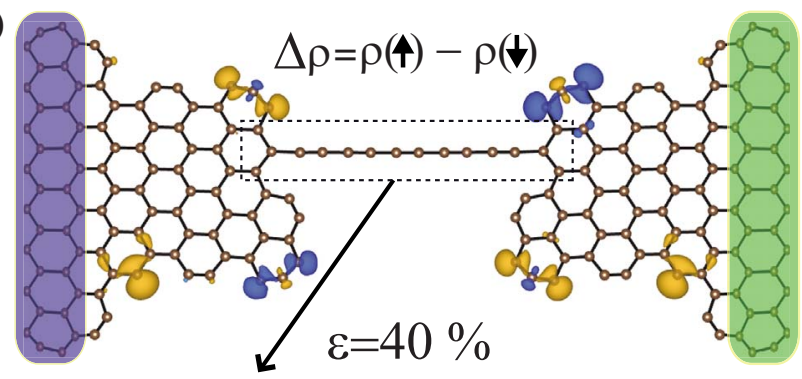

(d)

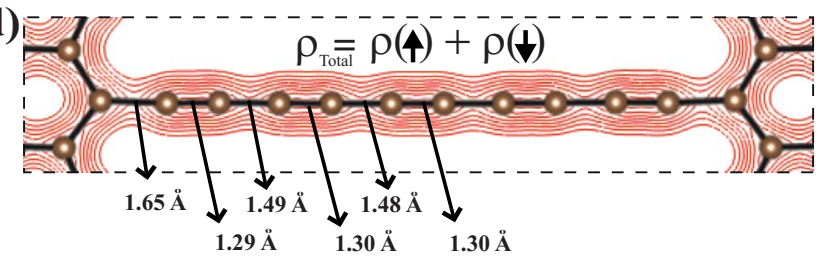

FIG. 2. (Color online) Stretching of a finite-size segment of the bare armchair graphene NR $\left(N_{A}=10\right)$ between two tapered ends. (a) The atomic structure of the NR corresponding to $\epsilon=0$ and $\epsilon=0.17$. (b) In the course of stretching, a hexagon connected to the chain is transformed to a pentagon by yielding a single carbon atom to the chain, which, in turn, becomes longer. In the left panel, one atom is incorporated in the chain when one bond of the hexagon is broken (c) A suspended chain comprising 12 carbon atoms is derived from graphene NR at $\epsilon=0.40$. The difference charge density of different spins states, $\Delta \rho(\uparrow, \downarrow)$ is also shown. (d) The chain structure with alternating short and long bonds are highlighted. The total charge density $\rho_{T}$ of both spins is denser around short C-C bonds. 

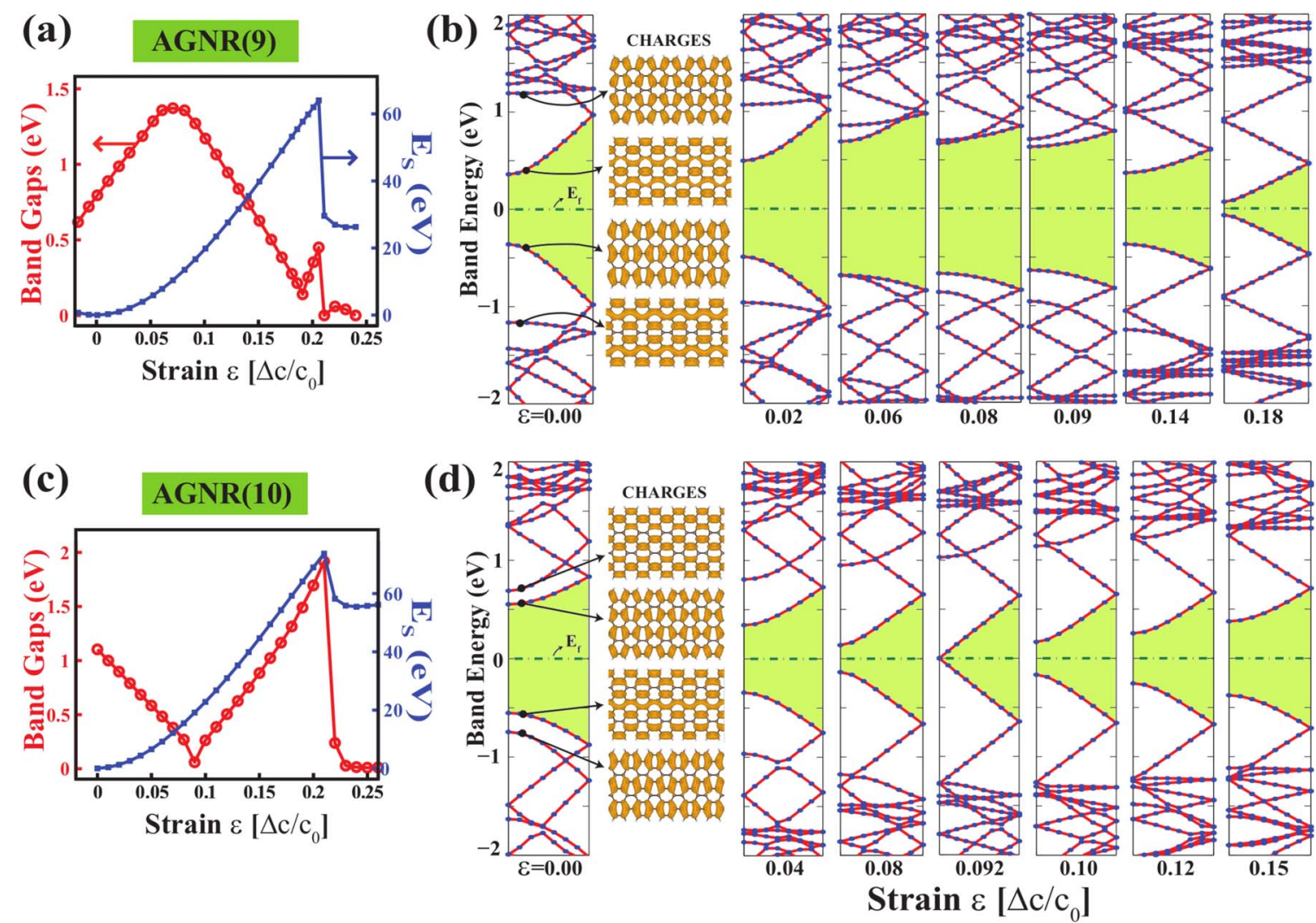

FIG. 3. (Color online) Variation in the energy band gaps of AGNR(9) and AGNR(10) with the strain from $\epsilon=0.0$ to $\epsilon=0.25$. (a) The band gap of AGNR(9) first increases with increasing strain in the elastic range, passes through a maximum, then decreases and eventually vanishes after the yielding point. (b) The band structures for different strain values and the isosurfaces of charge densities of lowest two conduction and highest two valance bands for zero-strain configuration. (c) Variation in the band gap of AGNR(10) with strain displays a reverse trend relative to $\operatorname{AGNR}(9)$. (d) Same as (b) for $\operatorname{AGNR}(10)$.

(MOFs). We also note that nonmagnetic armchair NRs attain spin-polarized (magnetic) ground state after they are broken into small pieces having zigzag edges. ${ }^{4}$ This is demonstrated by isosurfaces for the difference of spin-up and spin-down charge densities, $\Delta \rho(\uparrow, \downarrow)=\rho(\uparrow)-\rho(\downarrow)$ in Fig. 1 .

The behavior of a finite segment of bare armchair graphene NR under uniaxial tension between its two ends is also presented in Fig. 2(a). The tear, which initiates at one edge, propagates until the other edge and eventually the chain formation sets in. Usually triangles of atoms are formed at the region of junction of the nanoribbon pieces and the atomic chain. Upon stretching, the apex atom of the triangle is incorporated in the chain leaving behind a pentagonal (or broken hexagonal) ring as shown in Fig. 2(b). This way, a carbon atomic chain with alternating long and short bonds is suspended between two graphene pieces and grows by sequential implementation of atoms from these pieces to the chain. Here we point out an important difference between planar honeycomb NRs and metal nanorods both stretching in the plastic range. Experimental studies ${ }^{10}$ and theoretical simulations ${ }^{11}$ have demonstrated that metallic nanorods elongate in terms of sequential elastic and yielding stages. At the end of each elastic stage, the nanorod undergoes an orderdisorder transformation through the yielding stage resulting in a necking. Thus the onset of a subsequent elastic stage progresses through a smaller cross section. These structural changes were revealed by in situ measurements of two terminal conductance ${ }^{10}$ As for NRs here, the order-disorder transformation occurring at the end of each elastic stage and leading to necking is absent. Necking of a NR takes place through tearing. The transmission coefficient, $\boldsymbol{T}$ of carbon chain, which is calculated self-consistently using nonequilibrium Green's-function method reflects the combined electronic structure of central region and connecting electrodes. Results to be published elsewhere indicate that the implementation of a single carbon atom from hexagon to the chain is reflected to the variation in $\boldsymbol{T}$ under constant bias.

\section{VARIATION IN ELECTRONIC AND MAGNETIC PROPERTIES WITH STRAIN}

After massive structural changes taking place for $\epsilon>\epsilon_{Y}$, a NR attains new properties which are absent in its equilibrium state. For example, the ribbon achieves a higher chemical reactivity because of the unsaturated bonds protruding from atoms having lower coordination. Cumulene by itself is very reactive. Not only mechanical properties and atomic configuration but also the electronic and magnetic properties of nanoribbons can be modified through stretching as illustrated in Figs. 3 and 4. Depending on their widths, symmetries, and materials the band gaps of nanoribbons exhibit significant variations in the elastic deformation range, but usually they 
vanish in the plastic range. For example, variations in the band gaps of hydrogen saturated AGNR(9) and AGNR(10) with $\epsilon$ in the elastic range are rather different. The band gap of $\operatorname{AGNR}(9)$ is $\sim 0.7 \mathrm{eV}$ at $\epsilon=0.00$, but it increases with increasing strain up to $\epsilon=0.07$, but passes through a maximum and subsequently decreases to vanish at $\epsilon_{Y}$ as seen from Fig. 3(a). The band structure near the band gap and isosurfaces of charge densities of the lowest (highest) two states in the conduction (valence) band are presented in Fig. 3(b) for $\epsilon=0.00$. According to the electronic energy bands calculated as a function strain, while the highest valence band is lowered, the lowest conduction band is raised with increasing $\epsilon$. This, normally, increases the band gap. On the contrary, second highest (lowest) valence (conduction) band is raised (lowered) with increasing strain $\epsilon$. Consequently, the band gap of AGNR(9) first increases up to $\epsilon=0.07$, but decreases for $\epsilon>0.07$, where the second bands cross the first ones and dip in the gap. At the end, the orderings of the first and second valence and conduction bands are switched. In Figs. 3(c) and 3(d), the character and orbital compositions of the first and second valence and conduction bands are reversed in AGNR(10). Consequently, the variation in band gap with the strain is reversed in $\operatorname{AGNR}(10)$ relative to AGNR(9). While the highest (lowest) valence (conduction) band is raised (lowered), second valence (conduction) band is lowered (raised) and their dispersions is decreased with increasing $\epsilon$. This way the band gap decreases and is eventually closed within $3 \mathrm{meV}$ at $\epsilon \sim 0.092$. Even more remarkable is that the first conduction and valence bands, which are closed at $\mathbf{k} \rightarrow 0$, are linearized for $\mathbf{k}>0$. This means that the band energy has linear dispersion even for a small bias $\Delta V$; namely,

$$
E(\mathbf{k})-\left(E_{F} \pm \Delta V\right)=C \mathbf{k},
$$

where $C$ is a constant. Accordingly, hydrogen-terminated armchair nanoribbons AGNR(10), which is normally a semiconductor, behave like a 2D graphene and hence have carriers, i.e., holes or electrons, with massless Dirac fermion character at a specific value of $\epsilon$. This result is somehow unexpected but has important consequences: the massless Dirac fermion behavior in 2D graphene originating from linear band crossing at $K$ points at the Fermi level disappears when its size is finite. For example, massless Dirac fermion behavior is absent in an armchair nanoribbon, which is a nonmagnetic semiconductor at $\epsilon=0.00$. Whereas the massless Dirac fermion behavior would be highly desirable to achieve high carrier mobility in these nanoribbons. Realization of linearized bands as predicted in Fig. 3(b) is encouraging for various electronic applications, especially for fast nanoelectronics. We also note that different response of different bands to the tensile strain gives rise to a metalinsulator transition. For $\epsilon>0.09$, where the character and orbital composition of the highest valence and lowest conduction band switches, the band gap starts to open and to increase with increasing tensile strain, but vanishes suddenly at $\epsilon \sim \epsilon_{Y}$.

Because of zone folding, the character and the orbital composition of the bands at the edges of valence and conduction band are switched by going from $\operatorname{AGNR}(9)$ to
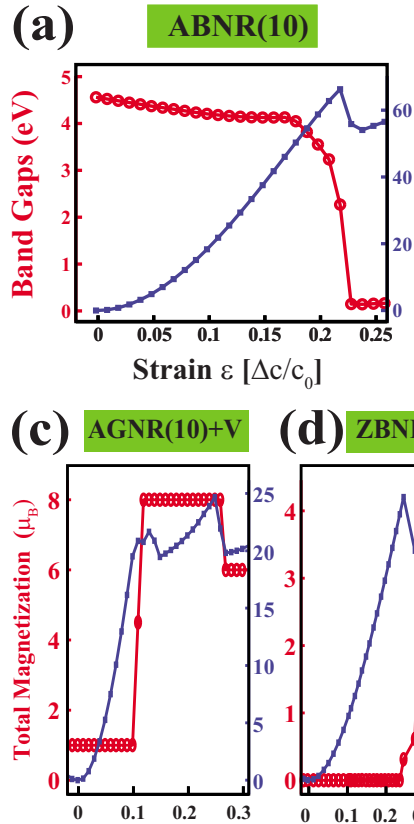

(c) $\operatorname{AGNR}(10)+V \quad$ (d) ZBNR(9)

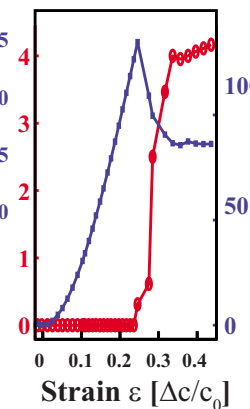

(b) ZBNR(9)

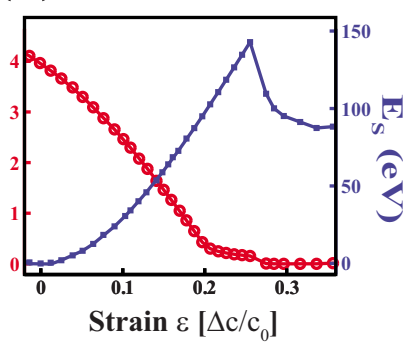

(e) $\operatorname{ZGNR}(9)$

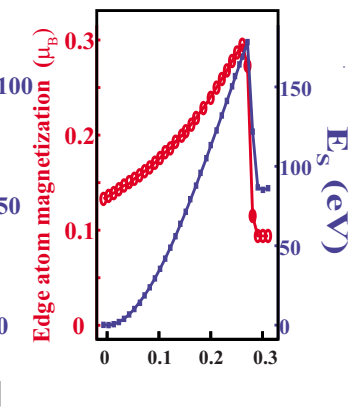

FIG. 4. (Color online) (a) Variation in the band gap of the armchair $\mathrm{BN}$ nanoribbon, $\mathrm{ABNR}(10)$, with strain $\epsilon$. (b) Zigzag $\mathrm{BN}$ nanoribbon ZBNR(10). (c) The magnetic moment of AGNR(10) having a single vacancy. $\mu$ jumps from $1 \mu_{B}$ to $8 \mu_{B}$ after the NR is torn suddenly from one edge. (d) Antiferromagnetic ZBNR(9) attains spin-polarized ground state after the yielding point. (e) Magnetic moment of a single edge atom of ZGNR(9) (which is antiferromagnetic in equilibrium) increases with strain in the elastic range and then falls suddenly at the yielding point.

AGNR(10). Therefore, similar effects can be observed in other families of AGNR having different widths, $N_{A}$. On the other hand, different response of different bands to the strain is an interplay between bond length and bonding (antibonding) bond energies. In fact, we distinguish two different bonds; namely, lateral (parallel to the ribbon axis) and tilted bonds, which have different response to $\epsilon$. As $\epsilon$ in the elastic range increases, the length of the tilted bonds in AGNR(9) first increase, passes a maximum and decreases reminiscent of the variation in the band gap in Fig. 3(a). The values of the strain corresponding to maximum of band gap and maximum length of tilted bonds are close. The elongation of the tilted bonds differ depending on their location relative to the edge. As for the lengths of the lateral bonds, they increase with increasing $\epsilon$, even if their elongations differ depending on their positions relative to the edge of the ribbon.

Figure 4 shows the variation in the band gaps and magnetic properties for other nanoribbons. In contrast to AGNR(10), the wide band gap of ABNR(10) in Fig. 4(a) does not change considerably for $0<\epsilon<0.20$ in the elastic range. However, the wide band gap of ZBNR(9) decreases steadily from 4 to $0.3 \mathrm{eV}$ between $0<\epsilon<0.20$ as shown in Fig. 4(b). As a result of plastic deformation either a spinpolarized state is induced or the existing magnetic state is modified. The magnetic moment of $1 \mu_{B}$ of $\operatorname{AGNR}(10)$ having a single carbon vacancy in a supercell of 5 unit cells increases to $8 \mu_{B}$ in the plastic range as shown in Fig. 4(c). 
This dramatic increase in the magnetic moment is attained by the severe modification of the honeycomb structure after the yielding point. Figure 4(d) shows that the antiferromangetic spin state of ZBNR(9) changes into a ferromagnetic state. The magnetization of edge atoms of zigzag graphene nanoribbon ZGNR(9) increases in the elastic range but falls to a lower value as shown Fig. 4(e). These results suggest that uniaxial strain can be used to monitor the electronic and magnetic properties of one-dimensional nanoribbons both in the elastic and plastic deformation ranges.

\section{CONCLUSIONS}

In this study the elastic constants of graphene, silicene, and $\mathrm{BN}$ zigzag and armchair nanoribbons are determined their unusual features are revealed under tensile stress. Their atomic, electronic, and magnetic structures are examined under elastic and plastic deformation range attained by adiabatic stretching. We found that in the course of elastic stretching, the electronic structure of these nanoribbons are strongly modified. The variation in band gap is sample and materials specific. For example, we showed that the variations in the band gaps of AGNR(9) and AGNR(10) with strain display reverse trends. The variation in band gap involves a complex interplay of zone folding, diverse elonga- tion of lateral and tilted $\mathrm{C}-\mathrm{C}$ bonds, and different orbital composition of the first and second valence and conduction bands. In particular, the closing of gap and linearization of highest and lowest conduction bands of a hydrogen saturated, armchair nanoribbon may have important implications, as such that massless Dirac fermion character can be realized even in semiconducting armchair nanoribbons. Unusual responses of band gaps to the strain are also obtained in different types nanoribbons.

The ending of elastic range and the onset of plastic deformation leading to diverse structural deformations and magnetic states in periodic and finite-size nanoribbons is another interesting outcome of this study. Structures having large holes are reminiscent of metal-organic frameworks, MOFs. We showed that long monatomic carbon chains can form in the course of stretching. Our prediction that suspended atomic chains can also be derived from $\mathrm{BN}$ and silicene nanoribbons under stretching in the plastic range is yet to be realized experimentally.

\section{ACKNOWLEDGMENTS}

Computing resources were partly provided by the $\mathrm{Na}$ tional Center for High Performance Computing of Turkey (UYBHM) under Grant No. 2-024-2007. This work is partially supported by TUBA, Academy of Science of Turkey.
${ }^{1}$ K. S. Novoselov, A. K. Geim, S. V. Morozov, D. Jiang, Y. Zhang, S. V. Dubonos, I. V. Grigorieva, and A. A. Firsov, Science 306, 666 (2004).

${ }^{2}$ Y. Zhang, Y.-W. Tan, H. L. Stormer, and P. Kim, Nature (London) 438, 201 (2005).

${ }^{3}$ K. S. Novoselov, A. K. Geim, S. V. Morozov, D. Jiang, M. I. Katsnelson, I. V. Grigorieva, S. V. Dubonos, and A. A. Firsov, Nature (London) 438, 197 (2005).

${ }^{4}$ E. H. Lieb, Phys. Rev. Lett. 62, 1201 (1989).

${ }^{5}$ S. Cahangirov, M. Topsakal, E. Akturk, H. Şahin, and S. Ciraci, Phys. Rev. Lett. 102, 236804 (2009).

${ }^{6}$ T. M. G. Mohiuddin, A. Lombardo, R. R. Nair, A. Bonetti, G. Savini, R. Jalil, N. Bonini, D. M. Basko, C. Galiotis, N. Marzari, K. S. Novoselov, A. K. Geim, and A. C. Ferrari, Phys. Rev. B 79, 205433 (2009).

${ }^{7}$ V. M. Pereira, A. H. Castro Neto, and N. M. R. Peres, Phys. Rev. B 80, 045401 (2009).

${ }^{8}$ V. M. Pereira and A. H. Castro Neto, Phys. Rev. Lett. 103, 046801 (2009).

${ }^{9}$ K. S. Kim, Y. Zhao, H. Jang, S. Y. Lee, J. M. Kim, K. S. Kim, J.-H. Ahn, P. Kim, J.-Y. Choi, and B. H. Hong, Nature (London) 457, 706 (2009).

${ }^{10}$ N. Agrait, G. Rubio, and S. Vieira, Phys. Rev. Lett. 74, 3995 (1995).

${ }^{11}$ H. Mehrez and S. Ciraci, Phys. Rev. B 56, 12632 (1997); M. R. Sorensen, M. Brandbyge, and K. W. Jacobsen, ibid. 57, 3283 (1998).
${ }^{12}$ S. Tongay, R. T. Senger, S. Dag, and S. Ciraci, Phys. Rev. Lett. 93, 136404 (2004).

${ }^{13}$ P. E. Blochl, Phys. Rev. B 50, 17953 (1994).

${ }^{14}$ J. P. Perdew, J. A. Chevary, S. H. Vosko, K. A. Jackson, M. R. Pederson, D. J. Singh, and C. Fiolhais, Phys. Rev. B 46, 6671 (1992).

${ }^{15}$ Y.-W. Son, M. L. Cohen, and S. G. Louie, Nature (London) 444, 347 (2006).

${ }^{16}$ The capacity of spin-polarized DFT and its comparison with other well-known methods can be found in a review by R. Zeller in Computational Nanoscience: Do It Yourself, NIC Series Vol. 31, edited by J. Grotendorst, S. Blugel, and D. Marx (John von Neumann Institute for Computing, Julich, 2006), pp. 419-445.

${ }^{17}$ G. Kresse and J. Hafner, Phys. Rev. B 47, 558 (1993).

${ }^{18}$ G. Kresse and J. Furthmuller, Phys. Rev. B 54, 11169 (1996).

${ }^{19}$ B. I. Yakobson, C. J. Brabec, and J. Bernholc, Phys. Rev. Lett. 76, 2511 (1996).

${ }^{20}$ C. D. Reddy, S. Rajendran, and K. M. Liew, Nanotechnology 17, 864 (2006).

${ }^{21}$ C. Lee, X. Wei, J. W. Kysar, and J. Hone, Science 321, 385 (2008).

${ }^{22}$ C. Jin, H. Lan, L. Peng, K. Suenaga, and S. Iijima, Phys. Rev. Lett. 102, 205501 (2009).

${ }^{23}$ M. Saito and Y. Okamoto, Phys. Rev. B 60, 8939 (1999).

${ }^{24}$ R. T. Senger, S. Tongay, S. Dag, E. Durgun, and S. Ciraci, Phys. Rev. B 71, 235406 (2005). 